\title{
An Overview of Rough-Hybrid Approaches in Image Processing
}

\author{
Aboul Ella Hassanien, Ajith Abraham, Senior Member, IEEE, James F. Peters, Member, IEEE, \\ and Gerald Schaefer, Member, IEEE
}

\begin{abstract}
Rough set theory offers a novel approach to manage uncertainty that has been used for the discovery of data dependencies, importance of features, patterns in sample data, feature space dimensionality reduction, and the classification of objects. Consequently, rough sets have been successfully employed for various image processing tasks including image segmentation, enhancement and classification. Nevertheless, while rough sets on their own provide a powerful technique, it is often the combination with other computational intelligence techniques that results in a truly effective approach. In this paper we show how rough sets have been combined with various other methodologies such as neural networks, wavelets, mathematical morphology, fuzzy sets, genetic algorithms, bayesian approaches, swarm optimization, and support vector machines in the image processing domain.
\end{abstract}

\section{INTRODUCTION}

Rough set theory, initially introduced by Zdzisław Pawlak during the early 1980s [1] and further developed over the last 25 years (see, e.g., [2], [3], [4], [5], [6]) provides an approach to approximation of sets that leads to useful forms of granular computing. The basic idea is to discover to what extent a given set of objects (e.g., pixel windows in an image) approximate another of set of objects of interest. Objects are compared by considering their descriptions [12]. Rough set theory offers a novel approach to manage uncertainty that has been used for the discovery of data dependencies, importance of features, patterns in sample data, feature space dimensionality reduction, and the classification of objects. One important result of rough set theory is the extractions of if-then rules from information tables. Such rules have the potential to reveal previously undiscovered patterns in sample data. In addition, rough set methods can be used to classify new samples based on what is already known. Unlike other computational intelligence techniques, rough set analysis requires no external parameters and uses only the information presented in given data. Rough set theory can be used to assess the completeness of sample data: if the data is incomplete, it will suggest that more information about the objects is needed to build a good classification model. On the other hand, if the data is complete, rough sets can determine the minimum number of features needed for classification. In

A.E. Hassanien is with the Information Technology Department, FCI, Cairo University, and the System Department, CBA, Kuwait University, Kuwait, email: abo@cba.edu.kw

A. Abraham is with the Center for Quantifiable Quality of Service in Communication Systems, Norwegian University of Science and Technology, Trondheim, Norway, email: ajith.abraham@ieee.org

J.F. Peters is with the Computational Intelligence Laboratory, Department of Electrical \& Computer Engineering, University of Manitoba, Winnipeg, Canada

G. Schaefer is with the School of Engineering and Applied Science, Aston University Birmingham, U.K. effect, the rough set approach leads to feature space dimensionality reduction. This is very important for applications where domain knowledge is very limited or data collection is very expensive and laborious, because it ensures the data collected is just sufficient to build a good classification model without sacrificing accuracy or wasting time and effort to gather extra information about the objects [7], [8], [9], [2].

In recent years, rough sets have been combined with various other computational intelligence techniques to provide more adaptive and more effective algorithms. In this paper, we set out to provide an overview of such roughhybrid approaches applied in the image processing domain [23], [25], [50]. In particular, we show how rough sets have been successfully combined with neural networks, wavelets, mathematical morphology, genetic algorithms, fuzzy sets, Bayesian theory, swarm intelligence, and support vector machines to provide more effective image processing algorithms.

\section{Rough Set: An Overview}

Rough set theory [44], [45], [52], [2] is a fairly new intelligent technique for managing uncertainty that is used for the discovery of data dependencies, to evaluate the importance of attributes, to discover patterns in data, to reduces redundancies, and to recognize and classify objects. Moreover, it is being used for the extraction of rules from databases where one advantage is the creation of readable if-then rules. Such rules have a potential to reveal previously undiscovered patterns in the data; furthermore, it also collectively functions as a classifier for unseen samples. Unlike other computational intelligence techniques, rough set analysis requires no external parameters and uses only the information presented in the given data. One of the nice features of rough set theory is that it can tell whether the data is complete or not based on the data itself. If the data is incomplete, it will suggest that more information about the objects is required. On the other hand, if the data is complete, rough sets are able to determine whether there are any redundancies in the data and find the minimum data needed for classification. This property of rough sets is very important for applications where domain knowledge is very limited or data collection is expensive/laborious because it makes sure the data collected is just sufficient to build a good classification model without sacrificing the accuracy or wasting time and effort to gather extra information about the objects [44], [45], [52], [2].

In rough set theory, sample objects of interest are usually represented by a table called an information table. Rows of an information table correspond to objects and columns 
correspond to object features. For a given set B of functions representing object features and a set of sample objects $\mathrm{X}$, an indiscernibility relation $\sim_{B}$ is a set of pairs $\left(x, x^{\prime}\right) \in X \times X$ such that $\mathrm{f}(\mathrm{x})=\mathrm{f}\left(\mathrm{x}^{\prime}\right)$ for all $f \in B$. The relation $\sim_{B}$ defines a quotient set $X / \sim_{B}$, i.e., a set of all classes in the partition of $X$ defined by $\sim_{B}$. Rough set theory identifies three approximation regions defined relative to $X / \sim_{B}$, namely, lower approximation, upper approximation and boundary. The lower approximation of a set $X$ contains all classes that are subsets of $X$, the upper approximation contains all classes with non-empty intersections with $X$, and the boundary is the set difference between the upper and lower approximations. A review of this basic material can be found in sources such as [44], [45], [52], [2], [29].

\section{ROUGH SET-WAVELETS APPROACHES IN IMAGE PROCESSING}

The wavelet transform decomposes a signal into shifted and scaled versions of a reference (mother) wavelet. It has been shown to have good properties of time and frequency localization and is robust to time varying signal analysis. The wavelet coefficients represent measures of similarity of the local shape of the signal to the mother wavelet under different shifts and scales [43]. Wavelets with their multiresolution property, have been proved to be effective in the integrating of coarse features and finer resolution details of source images to produce a good fused image.

$\mathrm{Li}$ et al. [55] present an approach to minimize the redundancy of structure existing in frame-based wavelet neural networks using rough set theory. The original structure of the wavelet network is obtained through a time-frequency analysis. Then the redundant nodes are eliminated in light of the dependency between the output of the network and nodes in the hidden layers to optimize the structure of the wavelet network. Simulation results proved the proposed method to be simple and effective.

To increase the efficiency of image segmentation and classification, a pre-processing stage should be considered in order to enhance the quality of the input images. For example, a median filter with PCNN [20] can be used to reduce noise in an image. It operates one pixel in the image at a time, and looks at its closest neighbors to decide whether or not it is representative of its surroundings. The basic structure of a rough wavelet approach is given in Figure 1. The algorithm works as follows: it firstly finds out the concrete position of the noised pixel according to the firing pattern, and then removes the noise from the image with a median filter. Initially the threshold of all of the neurons are set to 0 , and during the first iteration all the neurons are activated, or output a pulse, which means that all neurons receive the maximal linking input in the next iteration. So the proper set of the PCNN's parameters will make the neurons corresponding to noisy pixels with high intensity fire in its neighborhood at the second iteration, and according to the current firing pattern the concrete position of noisy pixels can be identified. Then the noised pixels can be removed with $3 \times 3$ median filter. The removal of noisy pixels with low

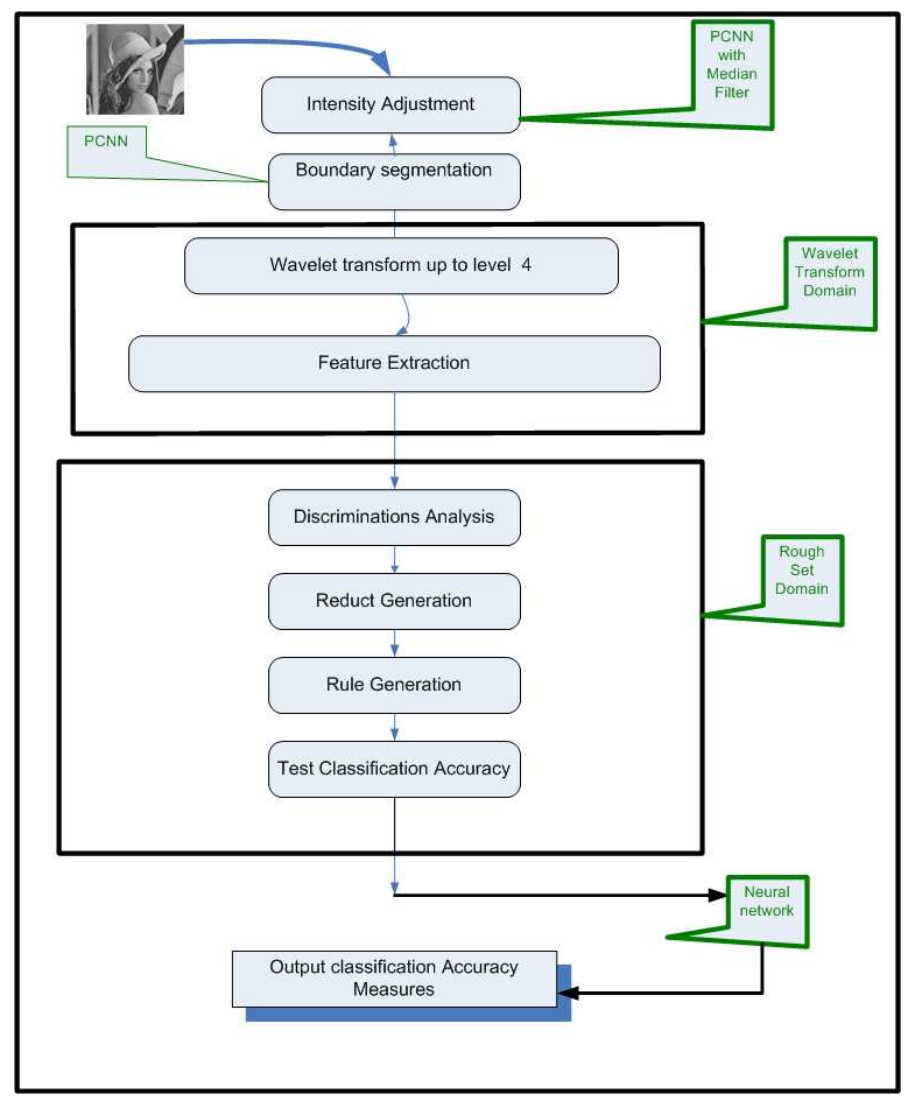

Fig. 1. Sample rough wavelet

intensity works similar with the intensities inverted. As the algorithm can locate the concrete positions of noisy pixels and apply the median operation only on these regions, it has the ability to preserve the details of the image.

Feature extraction plays an important role for classification as poorly implemented feature extraction or improper features will lead to poor classification results even when using the best possible classifier. Suitable features for classification can be created by combining wavelet theory with texture feature concepts based on the co-occurrence matrix [26]. The $N \times N$ input image will be decomposed row-wise for every row using a 1-D decomposition algorithm. The resulting two matrices are transposed and processed rowwise again to obtain four $\frac{N}{2} \times \frac{N}{2}$ square matrices. This procedure is repeated until a desired resolution is reached. The total number of coefficients after decomposition is always equal to the number of initial input coefficients. This matrix will be considered as a co-occurrence wavelet matrix. The produced wavelet coefficients yield a smaller set of more robust features, which can improve the probability of correct classifier. In order to reduce the size of the feature set, rough set analysis is applied to discover dependencies between the attributes and to generate a minimal set of attributes. The coefficients obtained by the rough set algorithm were used as inputs to a neural network to classify each image.

Another successful example introduced by Mingge et 
al. [54], which integrates rough sets and wavelet analysis for image fusion. Multifocus images are enhanced using rough sets, then fused using $\mathrm{Db} 4$ wavelets. The proposed algorithm is examined on images which are contaminated by salt-pepper noise. Entropy is used to evaluate image quality. Experimental results demonstrated the effectiveness of the proposed algorithm.

\section{ROUGH SET-NEURAL NETWORK APPROACHES IN IMAGE PROCESSING}

Neural networks are known for their ability to solve various complex problems in image processing. However, they are unable to determine redundant information from large data sets, which can easily lead to problems such as too complex network structures, long training times, and low converging speeds. Hassanien and Ślęzak [10] introduced a rough neural approach for rule generation and image classification. Hybridization of intelligent computing techniques has been applied to see their ability and accuracy to classify breast images into malignant and benign instances. Algorithms based on fuzzy image processing are first applied to enhance the contrast of the original image; to extract the region of interest and to enhance the edges surrounding that region. Then, features characterizing the underlying texture of the regions of interest are extracted using the greylevel co-occurrence matrix. A rough set approach to feature reduction and rule generation is then applied. Finally, a rough neural network is designed to discriminate different regions of interest in order to separate them into malignant and benign cases. The rough neural network employed is built from rough neurons [11], each of which can be viewed as a pair of sub-neurons, corresponding to the lower and upper bounds. Calculation of the input/output of the lower/upper rough neurons is performed as

$$
\begin{gathered}
\operatorname{Ir}_{L_{n}}=\sum_{j=1}^{n} w_{L_{n j}} O n_{j}, \\
\operatorname{Ir}_{U_{n}}=\sum_{j=1}^{n} w_{U_{n j}} O n_{j}, \\
O r_{L_{n}}=\min \left(f\left(\operatorname{Ir}_{L_{n}}\right), f\left(\operatorname{Ir}_{U_{n}}\right)\right), \\
O r_{U_{n}}=\max \left(f\left(\operatorname{Ir}_{L_{n}}\right), f\left(\operatorname{Ir}_{U_{n}}\right)\right) .
\end{gathered}
$$

where $\left(\operatorname{Ir}_{L_{n}}, O r_{L_{n}}\right)$ is the input/output of a lower rough neuron and $\left(\mathrm{Ir}_{U_{n}}, O r_{U_{n}}\right)$ the input/output of an upper rough neuron. The output of the rough neuron $\left(O_{r n}\right)$ is then computed as

$$
O_{r n}=\frac{O r_{U_{n}}-O r_{L_{n}}}{\operatorname{average}\left(O r_{U_{n}}, O r_{L_{n}}\right)} .
$$

The basic structure of a rough neural network is given in Figure 2. The introduced rough neural networks [11], [15], [13], [14] used, consist of one input layer, one output layer and one hidden layer. The number of hidden neurons

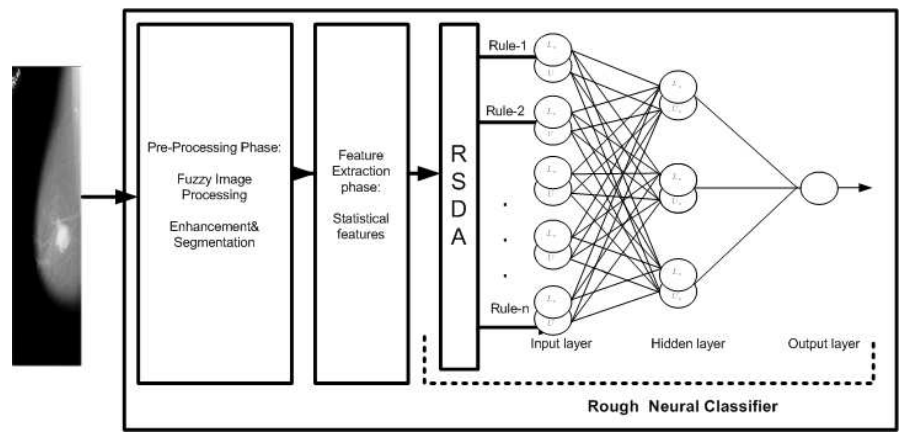

Fig. 2. Sample rough neural network [18]

is determined by [16], [17]:

$$
N_{h n} \leq \frac{N_{t s} * T_{e} * N_{f}}{N_{f}+N_{o}}
$$

where $N_{h n}$ is the number of hidden neurons, $N_{t s}$ is the number of training samples, $T_{e}$ is the tolerance error, $N_{f}$ is the number of features, and $N_{o}$ is the number of the output.

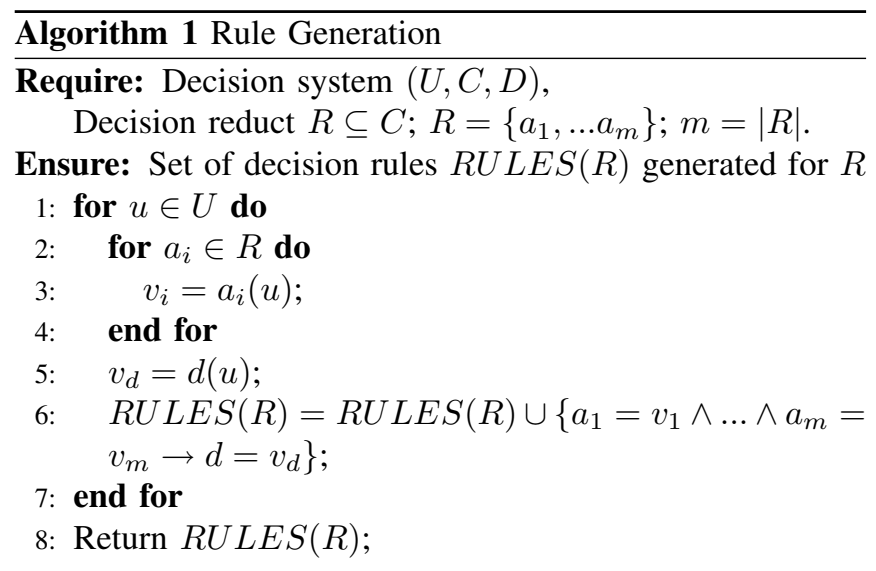

To evaluate performance of the presented rough neural approach, they run tests over different mammogram images. In their experiments, results show that the overall classification accuracy offered by rough neural approach is high compared with other intelligent techniques.

A rule importance measure $R_{I}$ was used as an evaluation to study the quality of the generated rule. It is defined by:

$$
R_{I}=\frac{\tau_{r}}{\rho_{r}}
$$

where $\tau_{r}$ is the number of times a rule appears in all reduct and $\rho_{r}$ is the number of reduct sets.

The quality of rules is related to the corresponding reduct(s) which are generating rules that cover the largest parts of the universe $U$. Covering $U$ with more general rules implies smaller size of a rule set. Importance rule criteria introduced in [30] were used to study the rules' importance [21].

Another successful example introduced by Jiang et al. [27], which integrates neural network with reduction based on rough set theory (which they called the rough neural 
network (RNN)) was used to classify digital mammograms. The experimental results showed that the RNN performs better than purely using neural network not only in terms of complexity, but also that it achieves a $92.37 \%$ classification accuracy compared to the $81.25 \%$ achieved using a normal neural network only.

Swiniarski and Hargis [28] described an application of rough sets methods to feature selection and reduction as a front end of neural-network-based texture images recognition. They applied include singular-value decomposition (SVD) for feature extraction, principal components analysis (PCA) for feature projection and reduction, and rough sets methods for feature selection and reduction. For texture classification a feedforward backpropagation neural network was employed. The numerical experiments showed the ability of rough sets to select reduced set of pattern's features, while providing better generalization of neural-network texture classifiers.

\section{ROUGH SET-MORPHOLOGY APPROACHES IN IMAGE PROCESSING}

Mathematical morphology [58], [59], [60] has many applications medical imaging, industrial inspection, computer vision, character recognition and others. It examines the geometrical structure of an image by probing it with small patterns, called structuring elements $E$, of varying size and shape, resulting in nonlinear image operators which are wellsuited to exploring geometrical and topological structures. A succession of such operators is applied to an image in order to make certain features apparent, distinguishing meaningful information from irrelevant distortions, by reducing it to a sort of skeletonization [24].

Mathematical morphology is a set algebra used to process and analyze data based on geometric shapes. The basic morphological operations are erosion and dilation. For binary signals, erosion is a Minkowski set subtraction and dilation a Minkowski set addition.

A review of these as well as other morphological operators can be found in [62]. The operators can be extended to operate on non-binary signals [62]. There are two main types of morphological filters. Set processing filters accept binary input signals and give binary output signals, while function processing filters accept binary or non-binary functions as input and yield non-binary functions as output. The interpretation of binary signals as sets and non-binary signals as functions is straightforward.

Bloch [57] illustrated that there is a tight relationship between rough set and mathematical morphology, that gives evidence of the increasing interest of the image analysis researchers towards these theories. Based on the observation that rough sets and mathematical morphology are both using dual operators sharing similar properties, Bloch [57] investigated more closely the links existing between both the domains. Author established the equivalence between some morphological operators and rough set defined from either a relation, or a pair of dual operators or a neighborhood system.
Recently, many successful works surrounding this issue has been addressed and discussed. For example, Yang et al. [56] proposed a method called membership-grade operator for binary image processing. Based on the same structural element, the operator achieves multiform dilations, erosions and edge extractions by proper choice of membership grade obtained by binary image. Applied to noise image, the operator can achieve the above processing and noise reduction, and good effect is obtained by image simulation. Skowron and Polkowski [38] proposed a method called analytical morphology for data filtering. The method was created on the basis of some ideas of rough set theory and mathematical morphology. Mathematical morphology makes an essential use of geometric structure of objects while the aim of their method is to provide tools for data filtering when there is no directly available geometric structure in the data set.

Complexity of morphological operations makes it desirable to propose a theoretical scheme of an approximate morphological calculus within a general paradigm of soft computing. Polkowski [37] proposed a scheme based on ideas of rough set. In his scheme, the underlying space of pixels is partitioned into disjoint classes by means of some primitive features and morphological operations are performed on classes, which allows for compression of image data. Author discuss topological foundation of morphology, in particulars spaces of rough fractals as well as morphological operations and he also point to plausible applications providing the approximate collage theorem.

Rough set was applied in spatial information theory to construct theories of granularity presenting information at different levels of detail. Stell [61] illustrated how by developing mathematical morphology in terms of relations we can obtain a framework, which includes the basic constructions of rough set theory as a special case.

Hassanien and Abraham [53] illustrated how rough set can be successfully integrated with mathematical morphology and provide a more effective hybrid approach to resolve medical imaging problems. Algorithms based on mathematical morphology are first applied to enhance the contrast of the whole original image; to extract the region of interest and to enhance the edges surrounding that region. Then, features are extracted characterizing the underlying texture of the regions of interest by using the gray-level co-occurrence matrix. The rough set approach to attribute reduction and rule generation is further presented. Finally, rough morphology is designed for discrimination of different regions of interest to test whether they represent malignant cancer or benign cancer. The experimental results illustrate that the overall performance in locating optimal orientation offered by the proposed approach is high compared with other hybrid systems such as rough-neural and rough-fuzzy systems.

A rough morphology approach is adopted for designing a hybrid system for rule generation and image classification. The architecture of the proposed hybrid approach is illustrated in Figure 3. It is comprised of four fundamental building phases: pre-processing, feature extraction, rough 


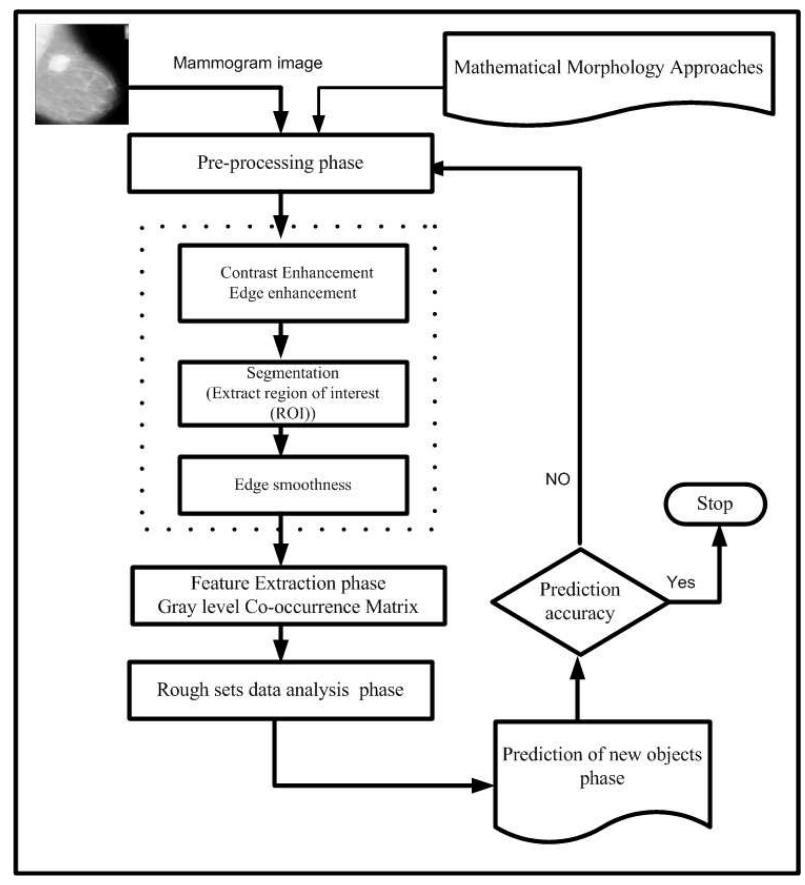

Fig. 3. Rough morphology hybrid approach [53]

set analysis and classification and prediction. In the first phase of the investigation, a pre-processing algorithm based on mathematical morphology is presented. It is adopted to improve the quality of the images and to make the feature extraction phase more reliable. Pre-processing stage involves enhancing mammogram images before a reasonable segmentation can be achieved as well as suppression noise in the segmented region of interest. Then, segmentation process involves grouping adjacent pixels with similar properties together to form connected regions. In the feature extraction phase, features extracted from the image regions are used to assign them to one of two classes: normal or abnormal. These stages have been added to provide a framework for the automatic analysis of the mammogram images, which is then evaluated using a pre-diagnosed image database. Rough set is used to classify the new image. The third phase is the rough set data analysis. It is done by computing the minimal number of necessary attributes, together with their significance, and generating the sets of rules. The last phase is the prediction of new objects, which is dependent on the type of generated rules and strategies based on the rough data analysis model. These four phases are described in detail in [53].

\section{ROUGH SET-FUZZY SET APPROACHES IN IMAGE PROCESSING}

Rough-fuzzy sets [31] can be seen as a particular case of fuzzy-rough sets. A rough-fuzzy set is a generalization of a rough set derived from the approximation of a fuzzy set in a crisp approximation space. This corresponds to the case where only the decision attribute values are fuzzy; the conditional values are crisp. The lower and upper approx- imations indicate the extent to which objects belong to a target set. Mao et al. [32] proposed a new fuzzy Hopfieldmodel net based on rough-set reasoning for the classification of multispectral images. The main purpose is to embed a rough-set learning scheme into the fuzzy Hopfield network to construct a classification system called a rough-fuzzy Hopfield net (RFHN). The classification system is a paradigm for the implementation of fuzzy logic and rough systems in neural network architecture. Instead of all the information in the image being fed into the neural network, the upper- and lower-bound grey levels, captured from a training vector in a multispectal image, are fed into a rough-fuzzy neuron in the RFHN. Therefore, only $2 / \mathrm{N}$ pixels are selected as the training samples if an N-dimensional multispectral image was used.

Wang et al. [33] proposed a new nearest neighbor clustering classification algorithm based on fuzzy-rough set theory (FRNNC). First, they make every training sample fuzzyroughness and use edit nearest neighbor algorithm to remove training sample points in class boundary or overlapping regions, and then use mountain clustering method to select representative cluster center points, then Fuzzy-Rough Nearest neighbor algorithm (FRNN) is applied to classify the test data. The new algorithm is applied to hand gesture image recognition, the results show that it is more effective and performs better than other nearest neighbor methods.

Hassanien [18] introduced a hybrid scheme that combines the advantages of fuzzy sets and rough sets in conjunction with statistical feature extraction techniques. The introduced scheme starts with fuzzy image processing as pre-processing technique to enhance the contrast of the whole image; to extracts the region of interest and then to enhance the edges surrounding the region of interest. Further, features from the segmented regions of the interested regions are extracted using the grey-level co-occurrence matrix. Rough set is used for generation of all reducts that contains minimal number of features and rules. Finally, these rules are passed to a classifier for discrimination for different regions of interest to classify images.

Image clustering analysis is one of the core techniques for image indexing, classification, identification and image segmentation. Mitra et al. [34] introduced a hybrid clustering architecture, in which several subsets of patterns can be processed together with an objective of finding a common structure. A detailed clustering algorithm is developed by integrating the advantages of both fuzzy sets and rough sets, and a measure of quantitative analysis of the experimental results is provided for synthetic and real-world data.

Petrosino et al. [35] presented a multi-scale method based on the hybrid notion of rough fuzzy sets, coming from the combination of two models of uncertainty like vagueness by handling rough sets and coarseness by handling fuzzy sets. Marrying both notions lead to consider, as instance, approximation of sets by means of similarity relations or fuzzy partitions. The most important features are extracted from the scale spaces by unsupervised cluster analysis, to successfully tackle image processing tasks. Mitra et al.'s [34] 
and Petrosino et al.'s [35] approach can be applied in many imaging clustering problems to obtain a compact representation of the image structures.

Sakar [36] generalizes the concept of rough membership functions in pattern classification tasks to rough-fuzzy membership functions and rough-fuzzy ownership functions. Unlike the rough membership value of a pattern, which is sensitive only toward the rough uncertainty associated with the pattern, the rough-fuzzy membership (or ownership) value of the pattern signifies the rough uncertainty as well as the fuzzy uncertainty associated with the pattern. Various set theoretic properties of the rough-fuzzy functions are exploited to characterize the concept of rough-fuzzy sets. These properties are also used to measure the rough-fuzzy uncertainty associated with the given output class.

\section{ROUGH SET-GENETIC ALGORITHM APPROACHES IN IMAGE PROCESSING}

Genetic algorithms and rough set theory have been used in combination in the study of images [22], [39], [40]. Lingras [39] proposed an unsupervised rough set classification using genetic algorithms (GS) and also illustrated how genetic algorithms can be used to develop rough sets. The proposed rough set theoretic genetic encoding are especially useful in unsupervised learning. A rough set genome consists of upper and lower bounds for sets in a partition. The partition may be as simple as the conventional expert class and its complement or a more general classification scheme.

Mitra et al. [40] described a way of designing a hybrid system for detecting the different stages of cervical cancer. Hybridization includes the evolution of knowledge-based subnetwork modules with Genetic Algorithm (GA) using rough set theory and the ID3 algorithm. Crude subnetworks for each module are initially obtained via rough set theory and the ID3 algorithm. These subnetworks are then combined, and the final network is evolved using genetic algorithms. The evolution uses a restricted mutation operator, which utilizes the knowledge of the modular structure, already generated, for faster convergence. The GA tunes the network weights and structure simultaneously.

\section{ROUGH SET-BAYESIAN APPROACHES IN IMAGE PROCESSING}

In many applications in computer vision and signal processing, it is necessary to assimilate data from multiple sources. For example in medical imaging, information on a patient may be available from a number of different modalities. The original rough set model is concerned primarily with algebraic properties of approximately defined sets. The Variable Precision Rough Set (VPRS) model extends the basic rough set theory to incorporate probabilistic information. As a result, there has been much recent research interest in this area. For example, Ślęzak et al. [41] presented a non-parametric modification of the VPRS model called the Bayesian rough set (BRS) model, where the set approximations are defined by using the prior probability as a reference. It is shown that the quality of BRS models can be

\begin{tabular}{|c|c|c|c|c|}
\hline \multirow{2}{*}{ Image } & \multirow{2}{*}{$\begin{array}{c}\text { Number of } \\
\text { Classes }\end{array}$} & \multicolumn{3}{|c|}{$\begin{array}{l}\text { Mean and standard deviation of the } \\
\text { DB index over the final clustering } \\
\text { results of } 25 \text { independent runs }\end{array}$} \\
\hline & & MEPSO & FVGA & FCM \\
\hline \multirow[b]{2}{*}{ IRS image of Mumbai } & \multirow[b]{2}{*}{$c=6$} & $\begin{array}{c}0.7283 \\
(0.0001)\end{array}$ & $\begin{array}{c}0.7902 \\
(0.0948)\end{array}$ & $\begin{array}{c}0.7937 \\
(0.0013)\end{array}$ \\
\hline & & $\begin{array}{c}2.6631 \\
(0.0018)\end{array}$ & $\begin{array}{l}2.1193 \\
(0.0826)\end{array}$ & $\begin{array}{l}2.1085 \\
(0.0043)\end{array}$ \\
\hline \multirow{2}{*}{$\begin{array}{l}\text { The Nomadic Super } \\
\text { Scout II Robot }\end{array}$} & \multirow[b]{2}{*}{$\mathrm{c}=4$} & $\begin{array}{c}0.2261 \\
(0.0017)\end{array}$ & $\begin{array}{c}0.2919 \\
(0.0583)\end{array}$ & $\begin{array}{r}0.3002 \\
(0.0452)\end{array}$ \\
\hline & & $\begin{array}{c}0.1837 \\
(0.0062)\end{array}$ & $\begin{array}{c}0.1922 \\
(0.0096)\end{array}$ & $\begin{array}{l}0.1939 \\
(0.0921)\end{array}$ \\
\hline
\end{tabular}

Fig. 4. Segmentation results for two real life grayscale images

evaluated using probabilistic gain function, which is suitable for identification and elimination of redundant features.

Swiniarski [42] described an application of rough sets and Bayesian inference for breast cancer detection using electropotentials. The statistical principal component analysis and the rough set methods were applied for feature extraction, reduction and selection. The quadratic discriminant was applied as a classifier for a breast cancer detection.

\section{ROUGH SET-SWARM INTELLIGENCE APPROACHES IN IMAGE PROCESSING}

Das et al. [46] hybridized rough set theory with Particle Swarm Optimization (PSO) algorithm. The hybrid roughPSO technique has been used for grouping the pixels of an image in its intensity space. Authors treated image segmentation as a clustering problem. Each cluster is modeled with a rough set. PSO is employed to tune the threshold and relative importance of upper and lower approximations of the rough sets. Davies-Bouldin clustering validity index is used as the fitness function, which is minimised while arriving at an optimal partitioning. Figure 4 (adapted from [46]) lists the value of DB index (and the corresponding number of clusters) calculated over the final solution on a test suite of two grayscale images using the rough-PSO hybrid algorithm (MEPSO), Evolutionary Fuzzy segmentation Algorithm (FVGA) and FCM.

Another approach that uses rough set with PSO has been proposed by Wang et al. [47]. The authors applied rough set to predict the degree of malignancy in brain glioma. As feature selection can improve the classification accuracy effectively, rough set feature selection algorithms are employed to select features. The selected feature subsets are used to generate decision rules for the classification task. A rough set attribute reduction algorithm that employs a search method based on PSO is proposed and compared with other rough set reduction algorithms. Experimental results show that reducts found by the proposed algorithm are more efficient and can generate decision rules with better classification performance. Moreover, the decision rules induced by rough set rule induction algorithm can reveal regular and interpretable patterns of the relations between glioma MRI features and the degree of malignancy, which are helpful for 


\section{CONCLUSIONS}

\section{ROUGH SET-SupPort VECTOR MACHINE APPROACHES IN IMAGE PROCESSING}

Support Vector Machines (SVMs) are a general algorithm based on guaranteed risk bounds of statistical learning theory. They have found numerous applications in image processing and pattern recognition and, in particular in medical imaging problems such as in classification of brain PET images, detection of microcalcification (MC) clusters in digital mammograms, lung cancer nodules extraction and classification, etc., and are now established as one of the standard computational intelligence tools. To inherit the merits of both Rough Set Theory (RST) and SVMs, a hybrid classifier called rough set support vector machines (RS-SVMs) is proposed by Gexiang et al. [48] to recognize radar emitter signals. RST is used as preprocessing step to improve the performances of SVMs. A large number of experimental results showed that RSSVMs achieve lower recognition error rates than SVMs and RS-SVMs have stronger capabilities of classification and generalization than SVMs, especially when the number of training samples is small.

Support vector machines (SVMs) are essentially binary classifiers. To improve their applicability, several methods have been suggested for extending SVMs for multiclassification, including one-versus-one (1-v-1), one-versusrest (1-v-r) and Decision Directed Acyclic Graph Support Vector Machines (DDAGGSVM). Lingras and Butz [49] described how binary classification with SVMs can be interpreted using rough sets and how rough set theory may help in reducing the storage requirements of the $1-\mathrm{v}-1$ approach in the operational phase. The introduced rough set approach to SVM classification removes the necessity of exact classification and is especially useful when dealing with noisy data. Next, by utilizing the boundary region in rough sets, they suggested two new approaches, extensions of (1$\mathrm{v}-\mathrm{r}$ ) and (1-v-1), to SVM multi-classification that allow for an error rate. Authors illustrated how the extended 1-v-r may shorten the training time and reduced storage requirements when compared to the conventional (1-v-r) approach.

Yun et al. [51] used a rough-support vector machine integration and developed the Improved Support Vector Machine (ISVM) algorithm to classify digital mammography images, where rough sets are applied to reduce the original feature sets and the support vector machine is used classify the reduced information.

Facial expression recognition is becoming more and more important in computer application, such as health care, children education, etc. Based on geometric feature and appearance feature, there are a few works been done on facial expression recognition using rough set and support vector machines. Chen et al. [19] proposed a novel approach based on rough set theory and SVM by considering only geometric features.
In this paper we provided an overview of different roughhybrid approaches applied in the image processing domain. While rough sets provide a powerful tool to describe uncertainties, which can be exploited in various image processing tasks the combination of rough sets with other computational intelligence techniques is able to provide a more effective approach. We have illustrated that rough sets have been successfully combined with fuzzy sets, wavelets, mathematical morphology, neural networks, genetic algorithms, support vector machines and swarm intelligence algorithms for tasks including image segmentation, feature extraction, classification and enhancement.

\section{REFERENCES}

[1] Z. Pawlak, Classification of Objects by Means of Attributes, Institute for Computer Science, Polish Academy of Sciences, Report 429, 1981.

[2] Z. Pawlak, "Rough sets", International J. Comp. Inform. Science, vol. 11, pp.341-356, 1982

[3] Z. Pawlak, "Rough classification", International Journal of ManMachine Studies, vol. 20, no. 5, pp. 469-483, 1984.

[4] Z. Pawlak, A. Skowron, "Rudiments of rough sets", Information Sciences, vol. 177, no. 1, pp. 3-27, 2007.

[5] Z. Pawlak, A. Skowron, "Rough sets: Some extensions", Information Sciences. An International Journal, Elsevier, vol. 177, no. 1, , pp. 28-40, 2007.

[6] Z. Pawlak, A. Skowron, "Rough sets and Boolean reasoning", Information Sciences. An International Journal, Elsevier, vol. 177, no. 1, pp. 41-73, 2007.

[7] Z. Pawlak, Rough Sets. Theoretical Aspects of Reasoning About Data, Kluwer, The Netherlands, 1991.

[8] Z. Pawlak, J. Grzymala-Busse, R. Slowinski, W. Ziarko, "Rough Sets", Communications of the ACM, vol.38, no.11, pp.88-95, 1995.

[9] L. Polkowski, Rough Sets. Mathematical Foundations, Physica-Verlag, Heidelberg, 2003.

[10] A.E. Hassanien, D. Selzak (2006) Rough Neural Intelligent Approach for Image Classification: A Case of Patients with Suspected Breast Cancer", International Journal of Hybrid Intelligent Systems, vol. 3, no. 4, pp. 205-218, 2006.

[11] P. Lingras, "Rough neural networks", in: Proc. of the 6th Int. Conf. on Information Processing and Management of Uncertainty in Knowledgebased Systems, Granada, Spain, pp. 1445-1450, 1996.

[12] J.F. Peters, "Classification of perceptual objects by means of features", International Journal of Information Technology and Intelligent Computing, in press.

[13] J.F. Peters, L. Han, S. Ramanna, "Rough Neural Computing in Signal Analysis", Computational Intelligence, vol.17, no.3, pp. 493-513, 2001.

[14] J.F. Peters, A. Skowron, L. Han, S. Ramanna, "Towards Rough Neural Computing Based on Rough Membership Functions: Theory and Application", In: RSCTC, LNAI, vol. 2005, pp. 611-618, 2000.

[15] L. Han, J.F. Peters, S. Ramanna, R. Zhai, "Classifying faults in high voltage power systems: A rough-fuzzy neural computational approach", Lecture Notes in Artificial Intelligence, vol. 1711, pp. 47-54, 1999.

[16] F. Chiang, R. Braun, "Intelligent Failure Domain Prediction in Complex Telecommunication Networks with Hybrid Rough Sets and Adaptive Neural Nets", in 3rd Int. Information and Telecommunication Technologies Symp., pp. 1-8, 2004.

[17] X. Hu, T.Y. Lin, J. Han, "A New Rough Sets Model Based on Database Systems", Fundamenta Informaticae, vol. 59, no. 2-3, pp.135$152,2004$.

[18] A.E. Hassanien, "Fuzzy-rough hybrid scheme for breast cancer detection", Image and Computer Vision Journal, Elsevier, vol. 25, Issue 2, pp. 172-183, 2007.

[19] P. Chen, G. Wang, Y. Yang and J. Zhou, "Facial Expression Recognition Based on Rough Set Theory and SVM" Lecture Notes in Computer Science, Springer Berlin / Heidelberg, Rough Sets and Knowledge Technology, Volume 4062/2006, pp.772-777, 2006 
[20] E. El-dahshan, A. Redi, A.E. Hassanien, K. Xiao, ”Accurate Detection of Prostate Boundary in Ultrasound Images Using Biologically-inspired Spiking Neural Network" International Symposium on Intelligent Siganl Processing and Communication Systems Proceeding 2007. Xiamen, China, pp.333-336.

[21] J. Bazan, H.S. Nguyen, S.H. Nguyen, P. Synak, J. Wróblewski, "Rough Set Algorithms in Classification Problem”. In: Polkowski, L., Tsumoto, S., Lin, T.Y. (eds.), Rough Set Methods and Applications. Physica Verlag, Berlin, pp. 49-88, 2000.

[22] M. Borkowski, J.F. Peters, "Matching 2D image segments with genetic algorithms and approximation spaces", Transactions on Rough Sets V, LNCS 4100, pp. 63-101, 2006.

[23] G.J. Dong, Y.S. Zhang and Y.H. Fan "Remote Sensing Image Classification Algorithm Based on Rough Set Theory",Advances in Soft Computing, Springer Berlin / Heidelberg, Volume 40/2007, pp. 846851,2007

[24] J.M. Ali and A.E. Hassanien, "Mathematical Morphology Approach for Enhancement Digital Mammography Images", IASTED, International Conference on Biomedical Engineering (BioMED2004) February 16-18, In Innsbruck, Austria, 2004.

[25] Z. Wu and D. Li, "A rough sets approach of hyperspectral image classification" Proceedings of SPIE - Volume 6043 MIPPR 2005: SAR and Multispectral Image Processing, Liangpei Zhang, Jianqing Zhang, Mingsheng Liao, Editors, 604310 (Nov. 3, 2005)

[26] A.E. Hassanien, "Pulse coupled Neural Network for Detection of Masses in Digital Mammogram”, Neural Network World Journal, Vol. 2/06, pp.129-141,2006

[27] J. Yun, L. Zhanhuai, W. Yong, Z. Longbo, "A Better Classifier Based on Rough Set and Neural Network for Medical Images", Proc. of the Sixth IEEE Int. Conf. on Data Mining, pp.853-857, 2006.

[28] R.W. Swiniarski, L. Hargis, "Rough sets as a front end of neuralnetworks texture classifiers", Neurocomputing, vol. 36(1-4), pp.85-102 2001.

[29] S. Ning, W. Ziarko, J. Hamilton and Cercone N, "Using Rough Sets as Tools for Knowledge Discovery". In: First International Conference on Knowledge Discovery and Data Mining KDD'95, U.M. Fayyad, R. Uthurusamy (eds.), Montreal, Que., Canada, AAAI, pp.263-268, 1995.

[30] J. Li, N. Cercone, "A Rough Set Based Model to Rank the Importance of Association Rules", LNAI, vol. 3462, pp.109-118, 2005.

[31] P. Srinivasan, M.E. Ruiz, D.H. Kraft, J. Chen, "Vocabulary mining for information retrieval: rough sets and fuzzy sets", Information Processing \& Management, vol. 37, no. 1, pp. 15-38, 1998.

[32] C.-W. Mao, S.-H. Liu, J.-S. Lin, "Classification of multispectral images through a rough-fuzzy neural network", Optical Engineering, vol. 43, Issue 1, pp. 103-112, 2004.

[33] X. Wang, J. Yang, X. Teng, N. Peng, "Fuzzy Classifiers Fuzzy Rough Set Based Nearest Neighbor Clustering Classification Algorithm", Lecture Notes in Computer Science, vol. 3613, pp. 370-373, 2005.

[34] S. Mitra, H. Banka, W. Pedrycz, "Rough-Fuzzy Collaborative Clustering Systems", IEEE Transactions on Man and Cybernetics, Part B, vol. 36, Issue 4, pp. 795-805, 2006.

[35] A. Petrosino, G. Salvi, "Rough fuzzy set based scale space transforms and their use in image analysis", Int. J. of Approximate Reasoning, vol. 41, Issue 2, pp. 212-228, 2006.

[36] M. Sarkar, "Rough-fuzzy functions in classification", Fuzzy Sets and Systems, vol. 132, Issue 3, pp. 353-369, 2002.

[37] L. Polkowski, "Approximate mathematical morphology: rough set approach" http://citeseer.ist.psu.edu/326941.html (pdf file)

[38] A. Skowron and L. Polkowski "Analytical Morphology: Mathematical Morphology of Decision Tables", Fundamenta Informaticae, vol. 27 Issue: $2 / 3$ p. $255-271,1996$.

[39] P. Lingras (2001) Unsupervised Rough Set Classification using GAs", Journal Of Intelligent Information Systems, vol. 16(3), pp. 215-228, 2001.

[40] P. Mitra, S. Mitra, S. Pal, "Evolutionary modular MLP with rough sets and ID3 algorithm for staging of cervical cancer", Neural Computing and Applications, vol. 10, no.1, pp. 67-76, 2001.

[41] D. Ślęzak, W. Ziarko, "The investigation of the Bayesian rough set model", Int J of Approximate Reasoning, Data Mining and Granular Computing, vol. 40, issues 1-2, pp. 81-91, 2005.

[42] R.W. Swiniarski, "Rough Sets and Bayesian Methods Applied to Cancer Detection", First Int. Conf. on Rough Sets and Current Trends in Computing, pp. 609-616, 1998.
[43] A.E. Hassanien and H. Own, "Multresolution Image Denosing based on Wavelet Transform" International Journal on Machine Graphics \& Vision, Vol. 10, No. 2, pp. 221-230, 2001.

[44] Z. Pawlak, "Rough sets - Theoretical aspects of reasoning about data". Kluwer, 1991.

[45] Z. Pawlak, J. Grzymala-Busse, R. Slowinski and W. Ziarko, "Rough Sets". Communications of the ACM, vol.38, no.11, pp.88-95, 1995.

[46] S. Das, A. Abraham, S.K. Sarkar, "A Hybrid Rough Set-Particle Swarm Algorithm for Image Pixel Classification", Proc. of the Sixth Int. Conf. on Hybrid Intelligent Systems, pp. 26-32, 2006.

[47] X. Wang, J. Yang, R. Jensen, X. Liu, "Rough set feature selection and rule induction for prediction of malignancy degree in brain glioma", Computer methods and programs in biomedicine, vol. 8, no. 3, pp.147156, 2006.

[48] G. Zhang , Z. Cao, Y. Gu, "A Hybrid Classifier Based on Rough Set Theory and Support Vector Machines", Lecture Notes in Computer Science, vol. 3613, pp.1287-1296, 2005.

[49] P. Lingras, C. Butz, "Rough set based 1-v-1 and 1-v-r approaches to support vector machine multi-classification", Information Sciences: an International Journal, vol. 177, Issue 18, pp. 3782-3798, 2007.

[50] B. Qiu, C.S. Xu, Q. Tian, "An Automatic Classification System Applied in Medical Images", IEEE Int. Conf. on Multimedia and Expo, pp.1045-1048, 2006.

[51] Y. Jiang, Z. Li, L. Zhang, P. Sun, "An Improved SVM Classifier for Medical Image Classification", in M. Kryszkiewicz et al., Eds., Int. Conf. on Rough Sets and Emerging Intelligent Systems Paradigms, LNAI, vol. 4585, pp. 764-773, 2007.

[52] L. Polkowski, "Rough Sets: Mathematical Foundations". PhysicaVerlag, 2003.

[53] A.E. Hassanien and A. Abraham "Rough Morphology Hybrid Approach for Mammography Image Classification and Prediction" International Journal of Computational Intelligence and Application World Scientific Publishing Company, 2008 (in press)

[54] X. Mingge, H. You, X. Huang and S. Feng, "Image fusion algorithm using rough sets theory and wavelet analysis", Proceeding of the 7th International Conference on Signal Processing. ICSP04, Volume 2, 31 Aug.-4 Sept. 2004, pp. 1041-1044.

[55] Y. Li, J. Shen, Z. Lu "Structure optimization of wavelet neural network using rough set theory" Proceedings of the 4th World Congress on Intelligent Control and Automation, Volume 1, 2002, pp.: 652-655.

[56] G.Q. Yang, L.H. Jiang and Y. Li., "Application of Rough Sets in Binary Morphology" International Conference on Machine Learning and Cybernetics, pp.3446-3449.

[57] I. Bloch "On links between mathematical morphology and rough sets". Pattern Recognition 33(9), pp. 1487-1496 2000.

[58] G. Matheron, "Random Sets and Integral Geometry". New York: Wiley, 1974

[59] J. Serra, "Image Analysis and Mathematical Morphology". vol.1.London, Academic press, 1982.

[60] J. Serra, "Image Analysis and Mathematical Morphology", vol. 2, Theoretical Advances,London, Academic, 1988.

[61] J. G. Stell "Relations in Mathematical Morphology with Applications to Graphs and Rough Sets" Lecture Notes in Computer Science, Volume 4736/2007 Spatial Information Theory, pp.438-454, 2007.

[62] P. Soille, "Morphological Image Analysis", Principles and Applications. Springer, 1999. 